\title{
High-Performance Temporary Adhesives for Wafer Bonding Applications
}

Rama Puligadda ${ }^{1}$, Sunil Pillalamarri ${ }^{1}$, Wenbin Hong ${ }^{1}$, Chad Brubaker ${ }^{2}$, Markus Wimplinger ${ }^{2}$, and Stefan Pargfrieder ${ }^{3}$

${ }^{1}$ Brewer Science, Inc., 2401 Brewer Drive, Rolla, MO, 65401

${ }^{2}$ EV Group, 7700 S. River Parkway, Tempe, AZ, 85284

${ }^{3}$ EV Group, E. Thallner GmbH, DI Erich Thallner Strasse 1, St. Florian, A-4782, Austria

\section{Abstract}

Myriad structures for stacking chips, power devices, smart cards, and thin substrates for processors have one thing in common: thin silicon. Wafer thinning will soon be an essential process step for most of the devices fabricated and packaged henceforth. The key driving forces for thinned wafers are improved heat dissipation, three-dimensional stacking, reduced electrical resistance, and substrate flexibility. Handling of thin and ultrathin substrates however is not trivial because of their fragility and tendency to warp and fold. The thinned substrates need to be supported during the backside grinding process and through subsequent processes such as lithography, deposition, etc. Using temporary adhesives to attach the processed device wafer to a rigid carrier wafer offers an efficient solution. The key requirements for such materials are ease of application, coating uniformity with minimal thickness variation across the wafer, good adhesion to a wide variety of surfaces, thermal stability in processes such as dielectric deposition and metallization, and ease of removal to allow high throughput. An additional requirement for these materials is stability in harsh chemical environments posed by processes such as etching and electroplating. Currently available materials meet only a subset of these requirements. None of them meet the requirement of high-temperature stability combined with ease of removal. We have developed adhesives that meet a wide range of post-thinning operating temperatures. Additionally, the materials are soluble in industry-accepted safe solvents and can be spin-applied to required thicknesses and uniformity. Above all, the coatings can be removed easily without Performance Temporary Adhesives for Wafer Bonding Applications," in Enabling Technologies for 3-D Integration, edited by Christopher A. Bower, Philip E. Garrou, Peter Ramm, and Kenji Takahashi (Mater. Res. Soc. Symp. Proc. 970, Warrendale, PA, 2007), paper no. 0970-Y04-09. Used by permission. 
leaving any residue. This paper reports on the development of a wide range of temporary adhesives that can be used in wafer thinning applications while applying both novel and conventional bonding and debonding methods.

\section{Introduction}

The wafer thinning process has attracted increased attention recently due to its promising application in device miniaturization and packaging. The key driving forces for thinned wafers are improved heat dissipation, three-dimensional stacking, reduced electrical resistance, and substrate flexibility. A reduction in wafer thickness combined with an increasing wafer diameter produces the tendency for the wafer to warp and fold, and thus creates a demand for new methods of wafer handling. The thinned substrates need to be supported during the backside grinding process and through the subsequent processes such as lithography, deposition, etc. Using temporary adhesives to bond the processed device wafer to a rigid carrier wafer offers an efficient solution, and is becoming increasingly important in both integrated circuit and MEMS applications, mainly due to its low cost, ease of processing, and adaptability [1-5].

The process for temporary wafer bonding is shown in Figure 1. The front side of the carrier wafer will be coated with the adhesive, and the wafer will undergo an initial bake to remove the solvent. The device wafer (possibly after the application of a protective layer) will be brought into contact with the adhesive-coated carrier wafer under vacuum and pressure. Adhesives for the bonding process must adhere to a variety of semiconductor substrates (for example, silicon, gallium arsenide, indium phosphide) and different metals, insulators, and dielectrics. The adhesive layer between device and carrier wafers provides the mechanical strength required for thin-wafer handling. Adhesives should possess adequate flow properties to 
flow into structures on the front side of the device wafer to provide good bonding quality. In addition, the adhesives must be easy to apply; have adequate mechanical strength, thermal stability, and chemical resistance; and exhibit minimal total thickness variation (TTV) across large wafers. Also, temporary wafer bonding requires the adhesive to be easily removed without damaging the features on the active side of the device wafer over a short debonding time to increase throughput. At present, only a limited number of adhesives are available for this type of application. However, most of them must be removed with a solvent and require a long debonding time, which significantly reduces throughput [2]. The tapes and waxes currently used for temporary wafer bonding have limited thermal stability at higher temperatures and can be used only up to $170^{\circ}-200^{\circ} \mathrm{C}[3,4]$.

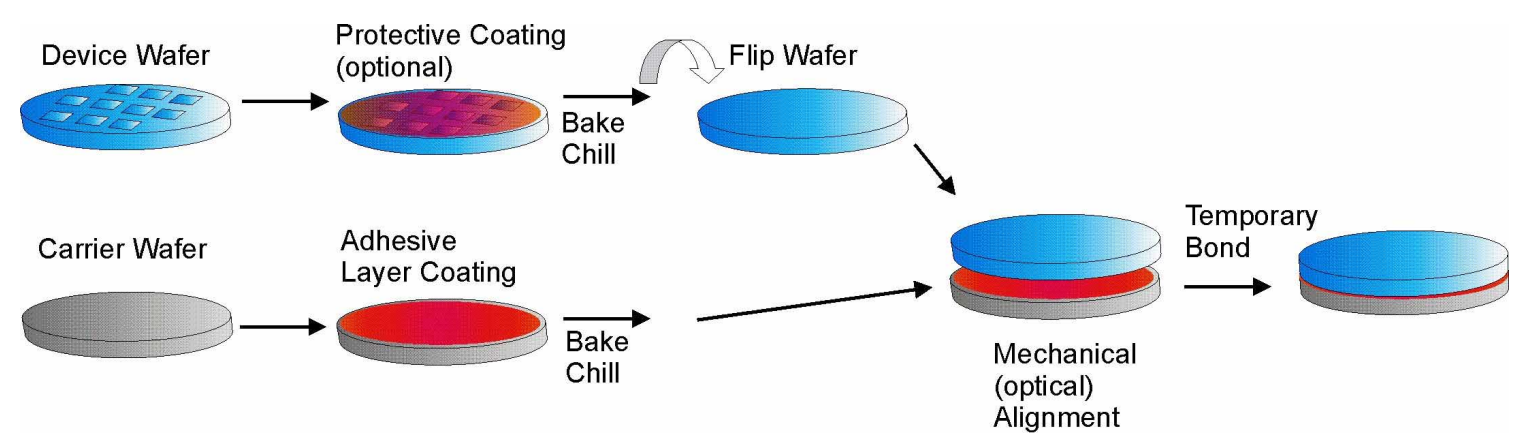

Figure 1. Temporary bonding process flow.

The objective of this work is to develop temporary adhesives for use in different waferthinning applications utilizing new methods for both bonding and debonding. We have developed a series of novel removable high-temperature spin-on adhesives with excellent bonding properties and a wide range of operating temperatures (from room temperature to $400^{\circ} \mathrm{C}$ ) for bonding and/or debonding to achieve a better processing window. The adhesives 
meet all the requirements for bonding strength, chemical resistance, thermal stability, and debonding time and temperature. The materials are soluble in safe solvents, can be spin-coated to obtain void-free thick films required for bump wafer applications, and achieve the required uniformity across the wafer. The resultant materials require a short time (less than 30 minutes) for adhesive removal and do not leave any residue after demounting. In short, the developed adhesives offer both time- and cost-efficiency.

\section{Materials}

Two different adhesive platforms have been developed based on different debonding mechanisms. Thermoplastic adhesives (I to IV in Table I) are thermally stable up to $200^{\circ} \mathrm{C}$ and can be debonded at temperatures ranging from $200^{\circ}$ to $250^{\circ} \mathrm{C}$ due to their relatively low viscosity ( $<100$ poise) at these temperatures. New removable themoset adhesives (I and II in Table I) have been designed for thermal stability above $300^{\circ} \mathrm{C}$. These spin-on materials undergo a thermal curing step to set the adhesives, and they can be debonded either by thermal decomposition above $350^{\circ} \mathrm{C}$ in nitrogen atmosphere or by exposure to light and heat. 
Table I. List of adhesives for temporary wafer bonding.

\begin{tabular}{|c|c|c|c|}
\hline \multicolumn{1}{c|}{ Sample } & Debonding method & $\begin{array}{c}\text { Debonding } \\
\text { temperature }\left(^{\circ} \mathbf{C}\right)\end{array}$ & $\begin{array}{c}\text { Maximum thickness } \\
\text { (microns) }\end{array}$ \\
\hline Thermoplastic I & Sliding & $200^{\circ}-250^{\circ}$ & 12.5 \\
\hline Thermoplastic II & Sliding & $200^{\circ}-250^{\circ}$ & 30 \\
\hline Thermoplastic III & Sliding & $200^{\circ}-250^{\circ}$ & 35 \\
\hline Thermoplastic IV & Sliding & $200^{\circ}-250^{\circ}$ & 40 \\
\hline Thermoset I & Thermal decomposition & $350^{\circ}-400^{\circ}$ & 10 \\
\hline Thermoset II & Thermal decomposition & $350^{\circ}-400^{\circ}$ & 10 \\
\hline
\end{tabular}

\section{Experimental Details}

The processing schemes for different types of adhesives shown in Table I for temporary wafer bonding are reported in Figure 2. The thermoset adhesives need at least two baking steps, one to remove the solvent and another to cure the polymer. Either glass or silicon carrier wafers could be used for thermoplastic and thermally decomposable adhesives. 

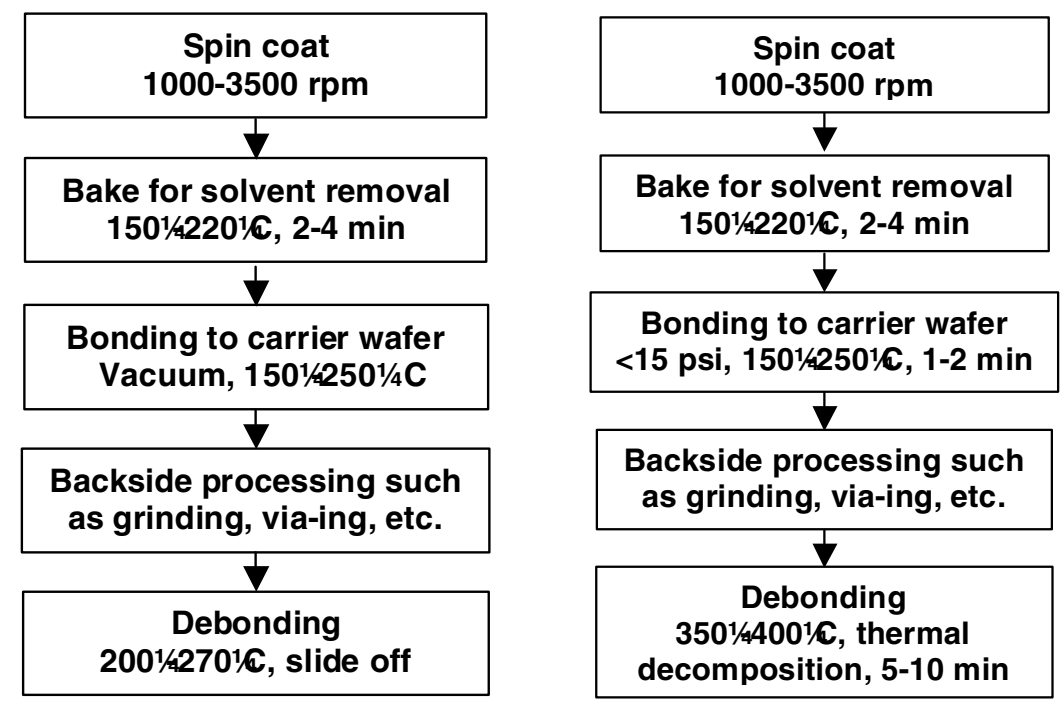

Figure 2. Process flow for temporary wafer bonding using removable adhesives thermoplastic (left) and thermoset (right)

It is worth mentioning that combining any of these processes together would be beneficial to reduce thermal cycling time in production. Because the very next step after solvent removal/soft bake of the adhesive film is to place it into the bonding system to undergo a thermocompression bonding process, placing the still-warm wafer into the bonding system directly saves time during the heating cycle of the bonding process. Because this will typically be the longest single step in the process, any process time reduction is favorable.

The $\mathrm{EVG}^{\circledR} 850$ Temporary Bonding System (Figure 3) has been designed to integrate the bonding/debonding steps. The modular design of the system allows inclusion of a coat module, a stack of bake modules (up to four), and two bonding modules, which provides a capability in excess of 30 bonded wafer pairs per hour. For experimental purposes, both 6- and 8-inch wafer pairs were processed using the $\mathrm{EVG}^{\circledR} 150$ resist processing system optimized for thick resist 
coating, and the $\mathrm{EVG}^{\circledR} 520 \mathrm{IS}$ wafer bonding system. Because of the modular nature of the system, the modules installed in the $\mathrm{EVG}^{\circledR} 850$ system are fully equivalent to the $\mathrm{EVG}^{\circledR} 150$ and $\mathrm{EVG}^{\circledR}$ 520IS systems.

The vacuum pressure during the bonding process is monitored to ensure that no outgassing of the adhesive occurs during bonding. The smooth increase of the vacuum level during the entire process confirms that no products were outgassing during bonding.

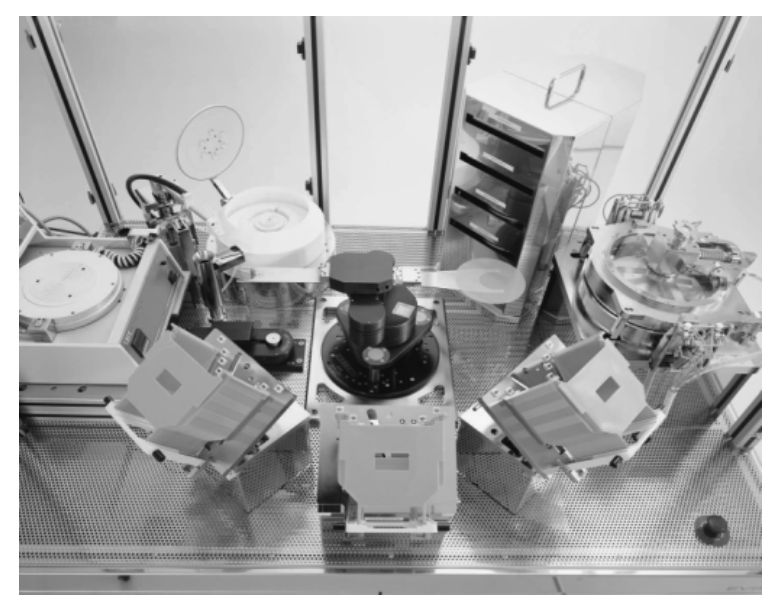

Figure 3. $\mathrm{EVG}^{\circledR} 850$ Temporary Bonding System.

\section{Results and Discussion}

Thickness measurements: The spin-on solutions offer better thickness control compared to waxes and tapes used in wafer bonding [4]. As the trend toward ultrathin wafers continues, it becomes very important for the adhesive to have a high degree of uniformity across the bond interface, as thickness variations cause breakage and warping during backgrinding. The thickness measurements on the adhesive films were carried out using an Alpha Step 200 (Tencor 
Instruments) and Nanospec ${ }^{\circledR} 6100$ (Nanometrics, Inc). Adhesives that can provide large thickness values will be required for bump wafer applications. The results of thickness measurements are shown in Figure 4. The film measured had a mean thickness of $13.5 \mu \mathrm{m}$ (at $1500 \mathrm{rpm}$ ), with a uniformity of $\pm 1.5 \%$. Therefore, the materials all lend themselves to very good uniformity control, which is crucial to effective bonding for backgrinding. In addition, the edge bead (another critical factor, since this bond is being performed across the full layer) was less than $2 \mu \mathrm{m}$ high and less than $0.5 \mathrm{~mm}$ wide.

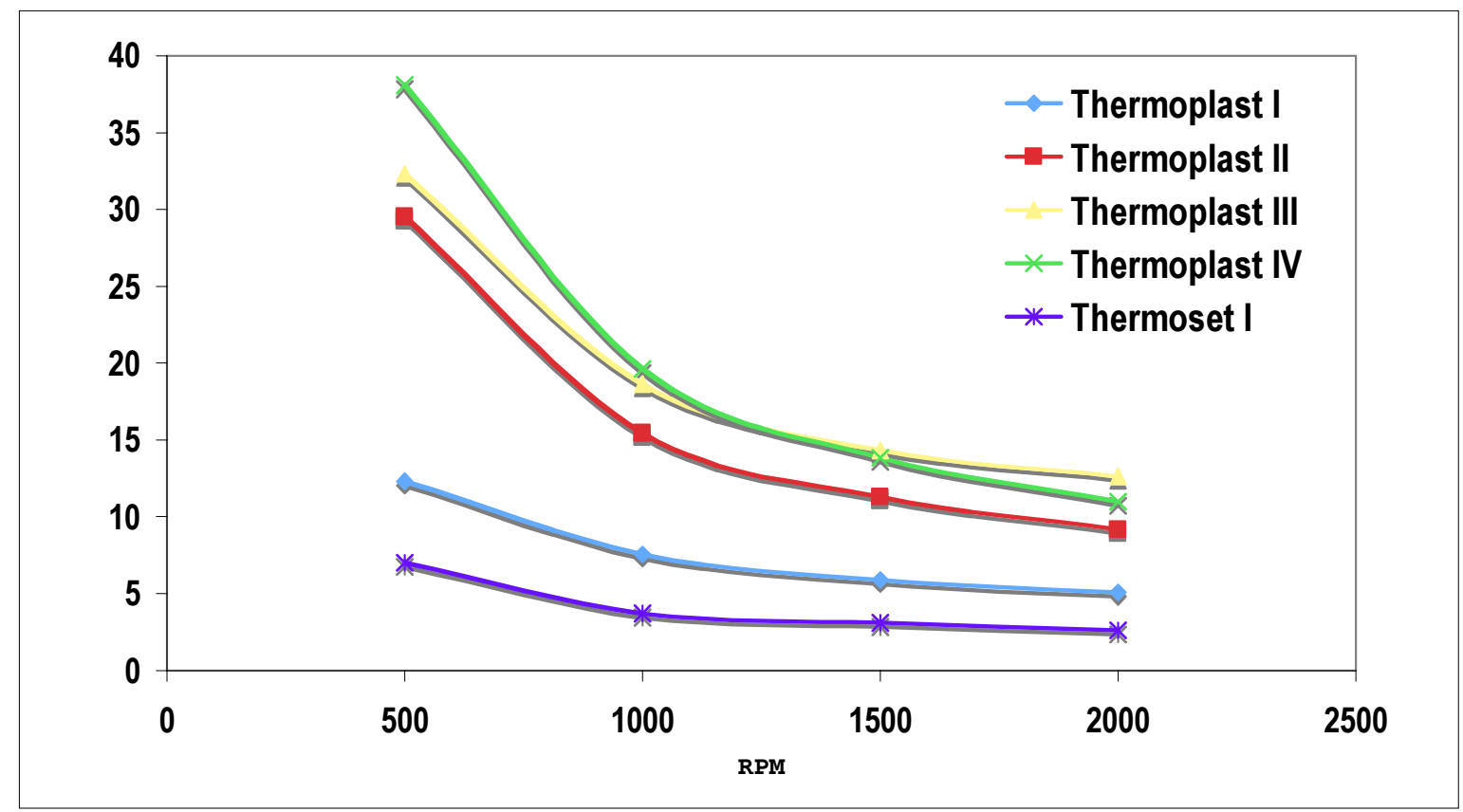

\begin{tabular}{|c|c|c|c|c|c|}
\hline \multirow[b]{2}{*}{ Sample } & \multicolumn{4}{|c|}{ Thickness $(\mu \mathrm{m})$} & \multirow[b]{2}{*}{ Avg TTV(\%) } \\
\hline & $\begin{array}{c}@ 500 \\
\text { rpm }\end{array}$ & $\begin{array}{c}@ 1000 \\
\text { rpm }\end{array}$ & $\begin{array}{c}@ 1500 \\
\text { rpm }\end{array}$ & $\begin{array}{c}\text { @ } 2000 \\
\text { rpm }\end{array}$ & \\
\hline Thermoplast I & 12.3 & 7.56 & 5.89 & 5.06 & 3.05 \\
\hline Thermoplast II & 38.08 & 19.64 & 13.83 & 10.99 & 4.46 \\
\hline Thermoplast III & 12.3 & 7.56 & 5.89 & 5.06 & 4.72 \\
\hline Thermoplast IV & 38.08 & 19.64 & 13.83 & 10.99 & 7.44 \\
\hline Thermoset I & 7.01 & 3.7 & 3.09 & 2.62 & 9.33 \\
\hline
\end{tabular}

Figure 4. Spin-speed and thickness data for the new adhesives. 
Chemical resistance: The adhesives intended for wafer bonding applications must withstand strong acids, alkalis, and solvents at high temperatures during various processes including etching and metallization. The summary of the resistance of the different adhesives to several chemicals is reported in Table II.

Table II. Summary of chemical resistance of the new adhesives.

\begin{tabular}{|c|c|c|c|}
\hline Sample & NMP & $\begin{array}{c}\mathbf{3 0 \%} \mathbf{~ K O H} \\
\left(\mathbf{8 5}^{\mathbf{0}} \mathbf{C}, \mathbf{6 0} \text { min }\right)\end{array}$ & $\mathbf{5 \%} \mathbf{H}_{\mathbf{2}} \mathbf{S O}_{\mathbf{4}}$ \\
\hline Thermoplastic I & Dissolves & No attack & Dissolves \\
\hline Thermoplastic II & No attack & No attack & No attack \\
\hline Thermoplastic III & No attack & No attack & No attack \\
\hline Thermoplastic IV & No attack & No attack & No attack \\
\hline Thermoset I & No attack & No attack & No attack \\
\hline Thermoset II & No attack & No attack & No attack \\
\hline
\end{tabular}

Optical transparency: All the adhesives have been designed to be transparent to provide the optical transparency required for wafer-to-wafer alignment.

Post-bonding uniformity: The coating thickness uniformity is extremely important, but only as it contributes to the final post-bonding uniformity, i.e., total thickness variation (TTV) of the bonded stack. The smaller the TTV, the more accurate and uniform the wafer thinning process 
becomes after the bonding. As substrates get thinner, TTV becomes more critical. If the TTV becomes a significant portion of the final wafer thickness (for instance, $15-\mu \mathrm{m}$ TTV versus 80 $\mu \mathrm{m}$ thickness), the performance consistency of the devices on the wafer is jeopardized.

To ensure good post-bonding uniformity, the pressure and temperature uniformity of the bonding system are critical. The resulting achieved TTV measurements in all cases were less than $10 \mu \mathrm{m}$ (Table III).

Table III. Post-bonding TTV values.

\begin{tabular}{|c|c|c|c|c|c|c|c|}
\hline & Adhesive & Left & Top & Center & Right & Botton & $\overline{\Gamma T V}$ \\
\hline & Thermoplastic & 1380 & 1378 & 1382 & 1378 & 1377 & 5 \\
\hline & Thermoplastic & 1380 & 1379 & 1382 & 1380 & 1380 & 3 \\
\hline & Thermoplastic & 1360 & 1357 & 1361 & 1358 & 1358 & 4 \\
\hline 3 & & & & & & & \\
\hline 6 & Thermoset I & 1377 & 1376 & 1376 & 1376 & 1376 & 1 \\
\hline & Thermoset I & 1376 & 1375 & 1377 & 1377 & 1377 & 2 \\
\hline & Thermoset I & 1378 & 1377 & 1376 & 1377 & 1375 & 3 \\
\hline & Thermoplastic & 1425 & 1426 & 1430 & 1425 & 1426 & 5 \\
\hline & Thermoplastic & 1421 & 1422 & 1428 & 1423 & 1426 & 7 \\
\hline 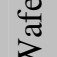 & Thermoplastic & 1433 & 1436 & 1439 & 1436 & 1434 & 6 \\
\hline$\infty$ & Thermoplastic & 1428 & 1428 & 1431 & 1424 & 1426 & 7 \\
\hline & Thermoplastic & 1394 & 1396 & 1399 & 1395 & 1395 & 5 \\
\hline
\end{tabular}


Adhesion strength: The adhesives should possess high bonding strength to support wafers during harsh processes such as backgrinding and polishing. The adhesion strength was evaluated using the peel test, the Maszara razor blade method [5], and rheological analysis.

The Maszara razor blade test is a method to determine the pull strength of the resulting bond. The test is performed by inserting a razor blade between the two bonded wafers, and then evaluating (via infrared for silicon wafers or visually for silicon-glass bonds) the length of the resulting crack. Figure 5 shows an image of a wafer pair bonded with the thermoset adhesive undergoing the crack test. Based on the thickness of the wafers and razor blade and on the length of the crack, the resulting bond force in this image is $\sim 1 \mathrm{~J} / \mathrm{m}^{2}$, which proved to be sufficient to withstand the backgrind processing subsequently performed. For the thermoplastic adhesives, the razor blade could not be inserted without breaking the wafers, which indicates that the bond strength is on the order of $2.5 \mathrm{~J} / \mathrm{m}^{2}$, the bulk fracture strength of silicon.

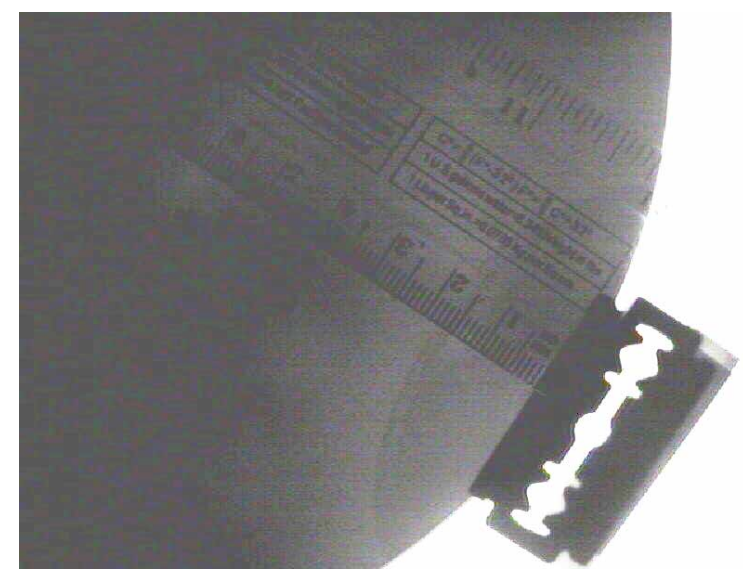

Figure 5. Maszara crack test, $15 \mathrm{~mm}$.

The results of rheological analyses are reported in Figure 6. The viscosities of the thermoplastic adhesives (Figure 6(a)) are less than 1000 poise at the debonding temperature, 
which makes it easy to slide apart the bonded wafer in the debonding step. On the other hand, the viscosities of the thermoset adhesives increase dramatically from $200^{\circ} \mathrm{C}$, indicating the generation of a crosslinked polymer network due to thermal curing. This behavior is expected from the material design.

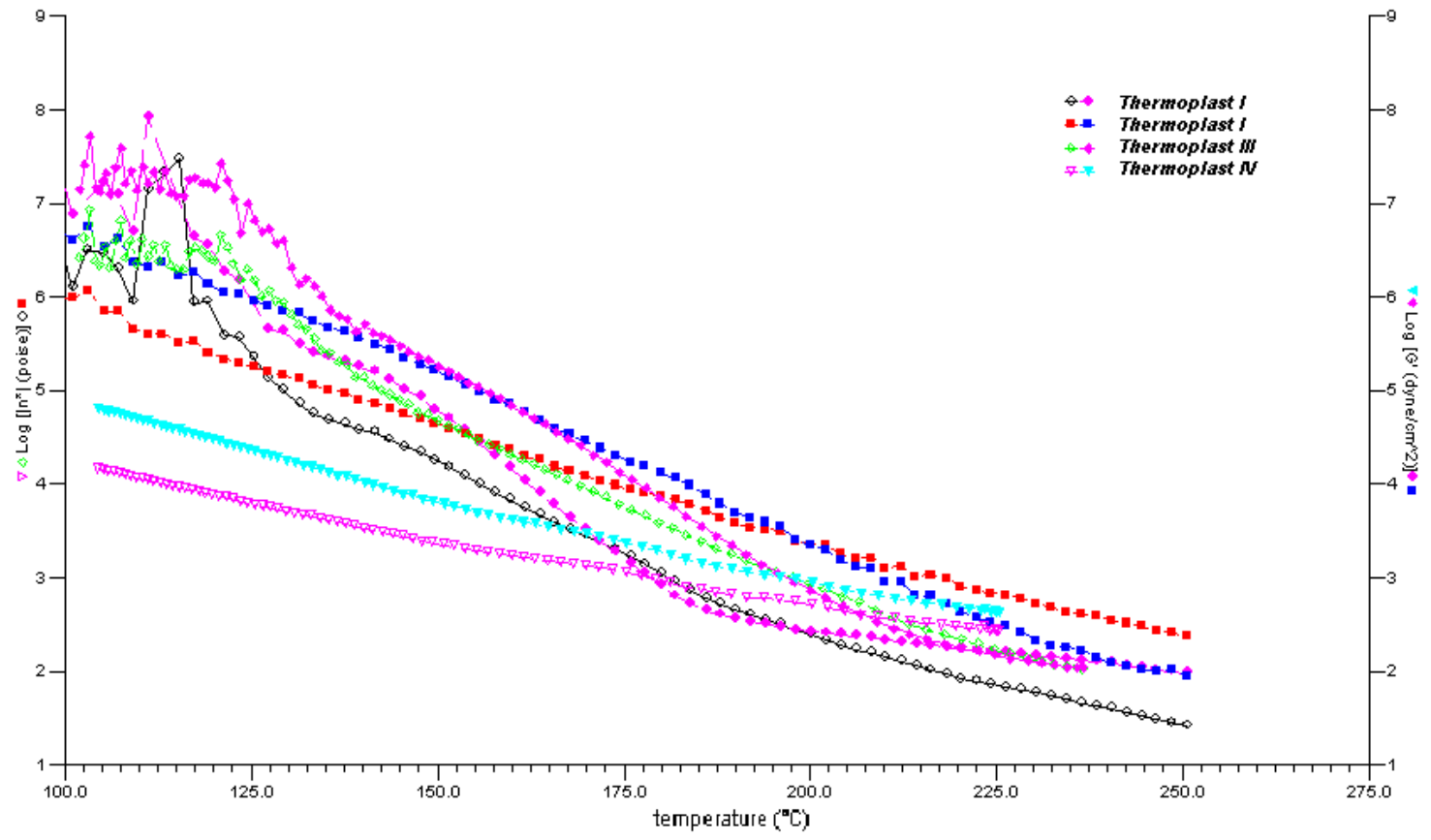

Figure 6(a). Rheological analysis of thermoplastic materials. 


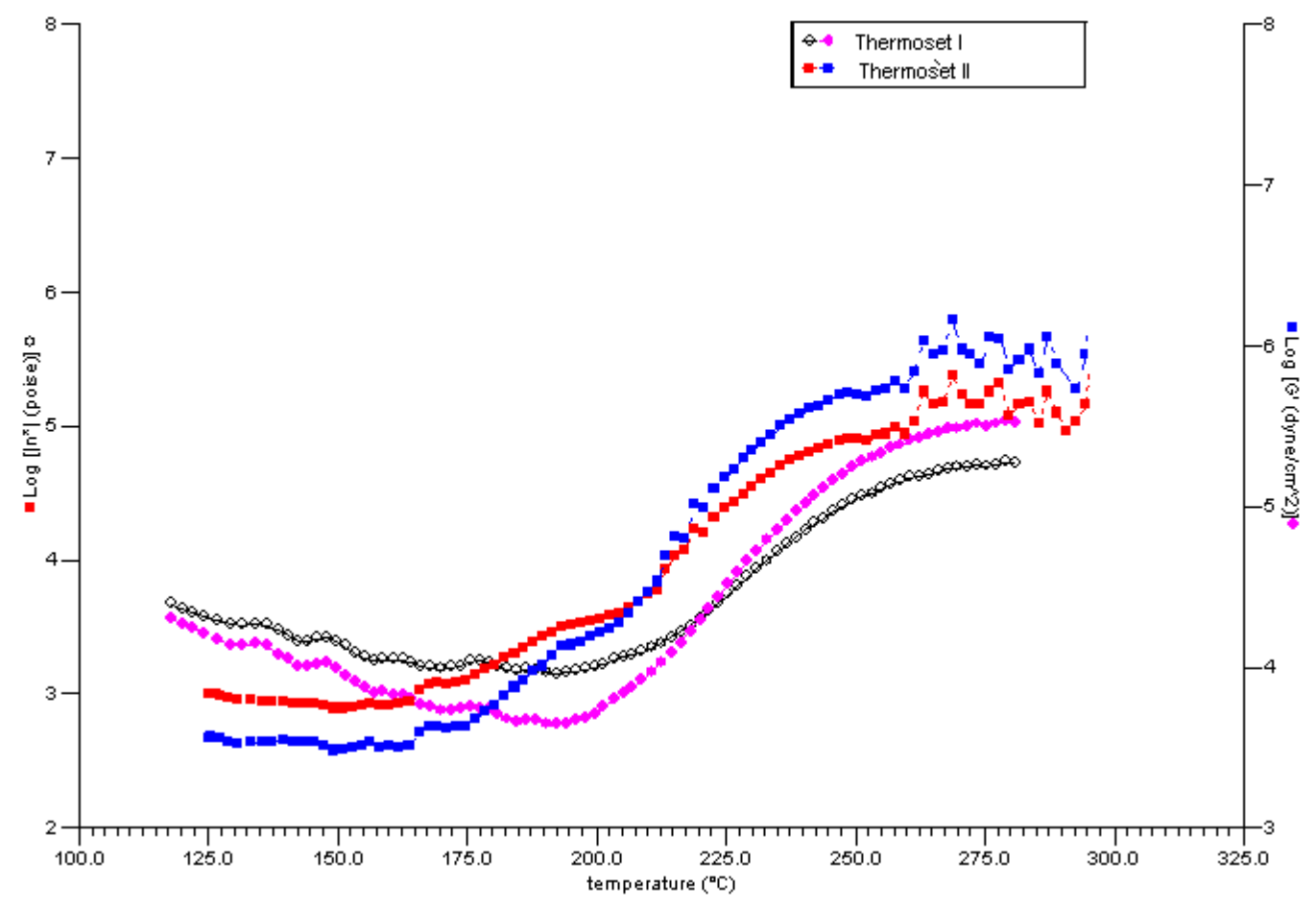

Figure 6(b). Rheological analysis of thermoset materials.

Void-free bonding: The final performance of the bonded pair relies not only on a good bonding uniformity in order to achieve good grinding performance. It also relies on achieving a strong and void-free bond. Even if the bond is very strong and very planar, voids, even very small ones ( 1 mm), can still cause issues in steps following backgrinding, such as metallization. Small voids such as this can burst under the conditions of the metallization process and leave defect points in the thinned substrate. However, by achieving a uniform resist coat and good pressure and temperature uniformity during the bond (plus bonding with sufficient force), voids can be eliminated. Figure 7 shows examples of acoustic wafer scans, performed with a SONIX Scanning Acoustic Microscope, which demonstrate the void-free bond capability. 


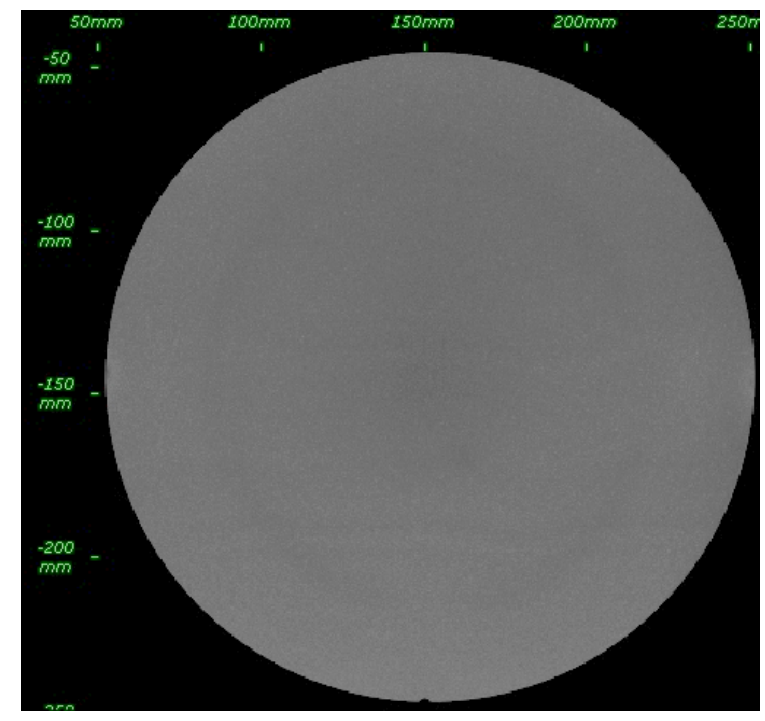

Figure 7. CSAM image of an 8-inch bonded wafer pair (thermoplastic material).

Back-grinding results: The image of a thinned wafer $(80 \mu \mathrm{m})$ using Thermoplastic I is shown in Figure 8(a). The back grinding was carried out using a combination of mechanical grinding and wet etching. Figure (8(b)) shows that the bond integrity was maintained through different backgrinding processes.

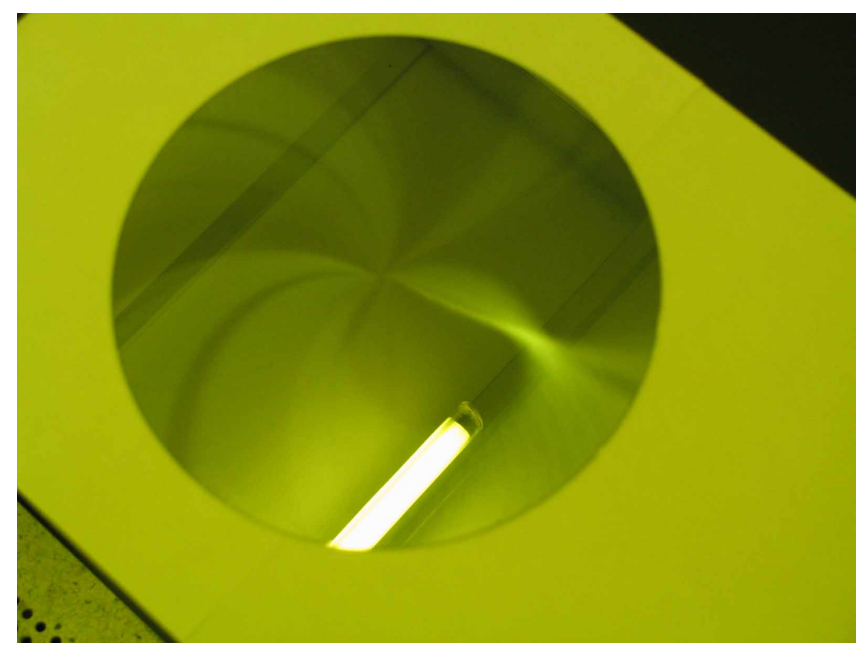

Figure 8(a). Image of an 8 -inch wafer thinned to $80 \mu \mathrm{m}$ using Thermoplastic I adhesive. 


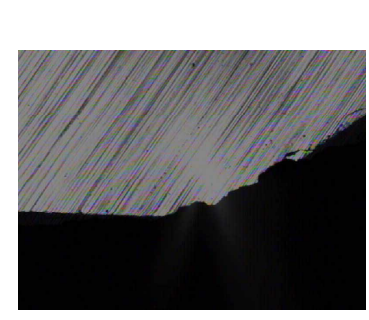

Notch ĞLeft 5X Objective

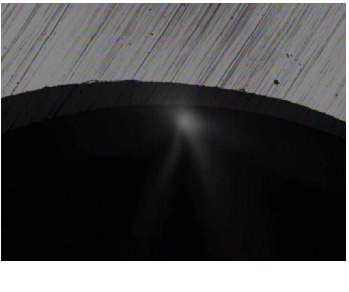

Notch Position 5X Objective

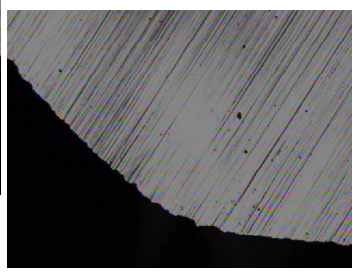

Notch ĞRight 5X Objective

Figure 8(b). Microscope images of wafer edges thinned to $80 \mu \mathrm{m}$ using Thermoplastic I adhesive.

Thermal properties: Due to the high processing temperatures at various stages of wafer thinning, it is very important for an adhesive to perform at a wide range of temperatures to avoid degradation and delamination. The thermal stability of the adhesives has been evaluated using thermogravimetric analysis (TGA). The thermoplastic materials are stable up to $200^{\circ} \mathrm{C}$. The reversible thermoset materials do not outgas until $275^{\circ} \mathrm{C}$. The thermograms of different adhesives are reported in Table IV.

Table IV. Isothermal TGA results of different adhesives.

\begin{tabular}{|c|c|c|}
\hline Sample & $\begin{array}{c}\text { \% Weight loss } \\
\left(\mathbf{2 0 0}^{\mathbf{}} \mathbf{C} / \mathbf{6 0} \mathbf{~ m i n}\right)\end{array}$ & $\begin{array}{c}\text { \% Weight loss } \\
\left(\mathbf{2 7 5}^{\mathbf{}} \mathbf{C} / \mathbf{6 0} \mathbf{~ m i n}\right)\end{array}$ \\
\hline Thermoplastic I & 0.23 & - \\
\hline Thermoplastic II & 2.6 & - \\
\hline Thermoplastic III & 1.88 & - \\
\hline Thermoplastic IV & 2.7 & 2.17 \\
\hline Thermoset I & - & 1.6 \\
\hline Thermoset II & - & - \\
\hline
\end{tabular}


Debonding and thinned wafer: As mentioned before, various methods can be used for debonding, depending on the adhesive (Figure 9).

For the thermoplastic adhesives, the debonding method involves heating the substrates to the debonding temperature (in most cases, typically above $200^{\circ} \mathrm{C}$ ) while they are locked into a rigid frame with top and bottom heaters. Then, the wafers are slid apart in a controlled manner such that the wafers stay parallel to each other until completely apart. Using two heaters is critical. If only one heater were used, then as the wafers separate, the influence of air would cause one substrate to cool, increasing the viscosity of the adhesive at the boundary between newly exposed surface and the still-covered surface. This situation would create a shear force variation, which can lead to wafer breakage.

The removable thermosets (Thermosets I and II) can be completely decomposed at temperatures above $350^{\circ} \mathrm{C}$ in nitrogen atmosphere. At $400^{\circ} \mathrm{C}$, the adhesives could be completely decomposed within 15 minutes without leaving any residue. Then the thinned wafer can be carefully removed from the carrier using a wedge-off motion.

All of these methods can be integrated into the $\mathrm{EVG}^{\circledR} 850 \mathrm{DB}$ debonding system for highvolume automated processing of these thin substrates. When all processes have been completed properly, the final result will be a completed and released thinned substrate suitable for final singulation and packaging. 


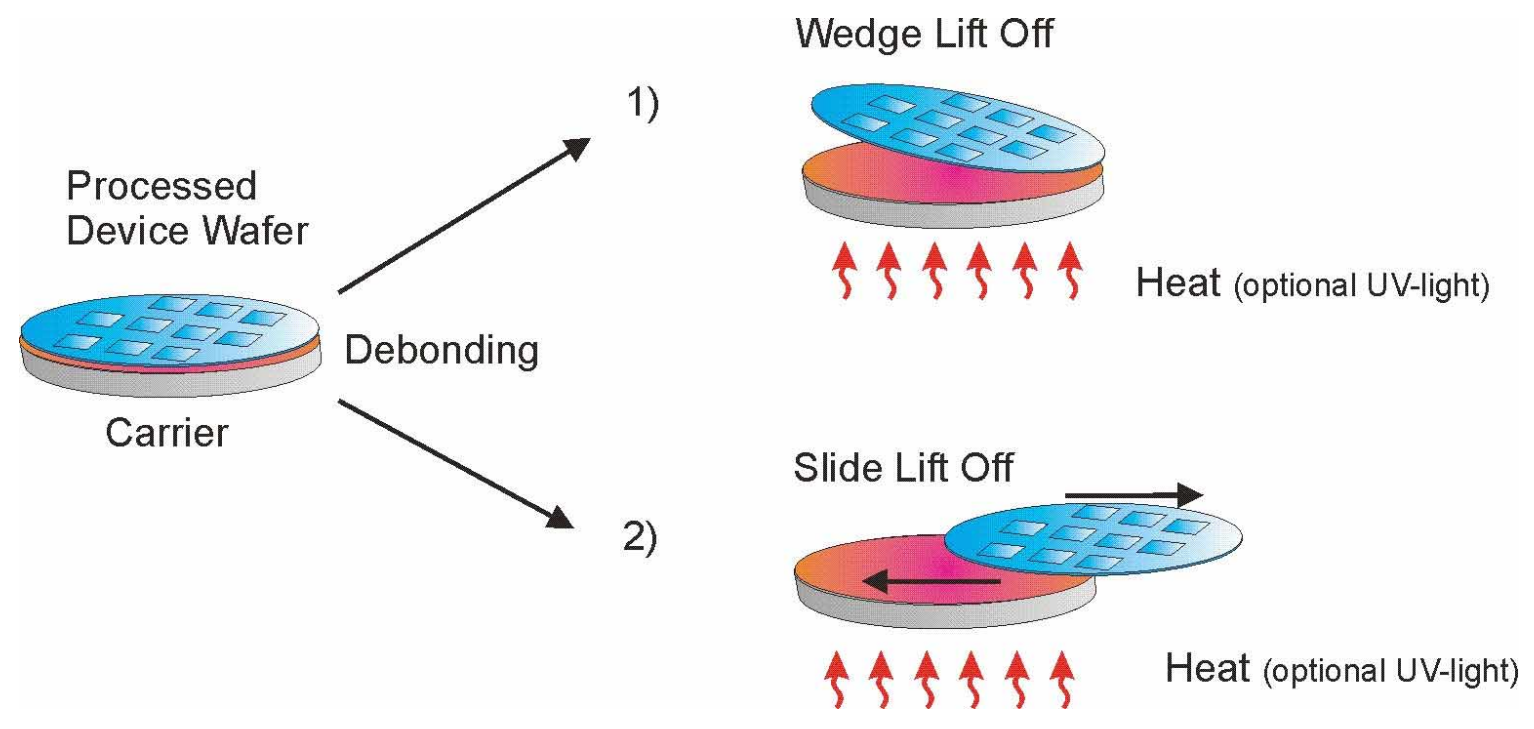

Figure 9. Debonding methods.

\section{Conclusions}

Two types of adhesives were evaluated for their potential use in temporary wafer bonding. The adhesives meet all the requirements for bonding strength, chemical resistance, thermal stability, and debonding times and temperatures. Of these, thermoplastic materials meet the requirements for $200^{\circ} \mathrm{C}$ applications. The materials are soluble in safe solvents, can be spincoated to obtain void-free thick films required for bump wafer applications, and achieve the required uniformity across the wafer. In addition, all the adhesives exhibit good chemical resistance and tunable debonding temperatures. The resultant materials require a short time for adhesive removal and do not leave any residue after demounting. In short, the developed adhesives offer both time- and cost-efficiency. 


\section{References}

1. F. Niklaus., G. Stemme, J.Q. Lu, “Adhesive wafer bonding,” J. Appl. Phys., 2006, 99, 031101.

2. J. Moore, A. Smith, D. Nguyen, S. Kulkarni, "High Temperature Resistant Adhesive for Wafer Thinning and Backside Processing," MANTECH 2004, 8.10.

3. C. Brubaker, M. Wimplinger, A. Malzer, P. Lindner, "Advances in Processing of Compound Semiconductor Substrates," MANTECH 2005, p. 261.

4. S. Combe, J. Cullen, M. O'Keefe. "Reversible Wafer Bonding: Challenges in Ramping up 150mm GaAs Wafer Production to Meet Growing Demand,” MANTECH 2006, p. 193.

5. Y. Kwon, J. Seok, "An evaluation process of polymeric adhesive wafer bonding for vertical system integration," Japanese Journal of Applied Physics, Part 1, vol. 44, no. 6A, 2005, pp. 3893-3902. 